\section{Case Reports in Oncology}

\title{
Endophytic-Type Endometrial Cancer with Adenomyosis Successfully Diagnosed with Hysteroscopic Endometrial Biopsy Using an 8.3-mm Operative Resectoscope: A Case Report
}

\author{
Michiko Honda ${ }^{a} \quad$ Akira Tsuchiya $^{a} \quad$ Wataru Isono ${ }^{a} \quad$ Mikiko Takahashi $^{b}$ \\ Akihisa Fujimoto $^{a}$ Masashi Kawamoto $^{b}$ Osamu Nishii ${ }^{a}$ \\ aDepartment of Obstetrics and Gynecology, University Hospital Mizonokuchi, Teikyo \\ University School of Medicine, Kawasaki, Japan; 'bepartment of Pathology, University \\ Hospital Mizonokuchi, Teikyo University School of Medicine, Kawasaki, Japan
}

\section{Keywords}

Endometrial neoplasms · Biopsy · Hysteroscopy

\begin{abstract}
In order to diagnose endometrial cancer preoperatively, outpatient endometrial biopsy with a curette is frequently performed owing to its convenience. However, in some cases, gynecologists fail to diagnose endometrial cancer via outpatient endometrial biopsy because of the cancer's distribution in the uterus and its consistency. A 57-year-old Japanese woman (gravida 4 para 4) presented with a 6 -month history of light but intermittent postmenopausal vaginal bleeding. A malignant uterine tumor was strongly suspected after imaging using ultrasound examination and magnetic resonance imaging; however, a precise pathological diagnosis was not achieved despite multiple outpatient endometrial biopsies with the aid of office hysteroscopy. Based on an endometrial biopsy obtained using a cutting loop electrode on an 8.3$\mathrm{mm}$ operative resectoscope, we reached a diagnosis of endophytic-type endometrial cancer, which is in accordance with the final pathological diagnosis after abdominal hysterectomy. Three months after her first visit to our hospital, total abdominal hysterectomy and bilateral
\end{abstract}


salpingo-oophorectomy with pelvic/para-aortic lymph node dissection were performed. Macroscopically, the endometrium was atrophic, and there was no obvious mass in the uterine cavity; however, microscopically, the cancer cells mainly existed in the deep myometrium and the final diagnosis was International Federation of Gynecology and Obstetrics (FIGO) stage IB endometrial cancer. Operative biopsy of the uterine endometrium and deep myometrium using hysteroscopy confirmed an accurate preoperative diagnosis of uterine endometrial cancer specifically of the endophytic type.

\section{Introduction}

The incidence and mortality rate of endometrial cancer (EC) in 2012 was $12.9-19.9$ and 0.9-3.8 per 100,000 women worldwide, respectively [1]. Between 2005 and 2011, the 5-year survival rate in the USA for localized EC was $95 \%$, and the prognosis for early-stage EC is favorable. However, when diagnosed at the advanced stage, EC patients can have a poor prognosis, with a 5-year survival rate of $68.3 \%$ for International Federation of Gynecology and Obstetrics (FIGO) stage III EC, and of $16.8 \%$ for FIGO stage IV EC [2,3]. Therefore, it is important to detect $\mathrm{EC}$ at an early stage if we are to improve the prognosis of these patients.

Since EC is typically found on the uterine endometrium and myometrial layer, outpatient endometrial biopsy using a sharp curette without anesthesia or dilatation is a convenient and low-cost procedure with approximately $90 \%$ sampling adequacy and pathological results $[4$, 5]. The high sensitivity and specificity rates of office-based endometrial biopsy for the detection of EC are well-known. However, there are some cases in which gynecologists fail to diagnose EC via an outpatient endometrial biopsy. Several risk factors associated with biopsy failure have been reported [5]. In addition to patients' postmenopausal status and atrophic endometrium, there are some rare cases in which the growth form and the consistency of endometrial tumor make an accurate diagnosis more difficult because the outpatient endometrial biopsy technique using a sharp curette cannot acquire specimens from the deep myometrium or specimens with a hard-enough consistency [6,7]. When a differential diagnosis of endophytic-type endometrial tumor is required, hysteroscopic resectoscope-directed biopsy under anesthesia would be useful. This is because the depth of a biopsy is needed to conduct adequate histologic evaluation of the endometrium and adjacent myometrial lesion, which includes tissue that is highly representative of endometrial tumor [8], and a 5-mm-thick tissue biopsy with a loop electrode could obtain a surgical specimen that satisfies pathologist's requirements for sufficient evaluation of endophytic-type endometrial tumor.

Here, we report a rare case of a postmenopausal woman with an endophytic-type endometrial adenocarcinoma co-existing with uterine adenomyosis. In this case, the definitive diagnosis was achieved with an operative biopsy using a hysteroscopic resectoscope after 2 failed attempts using outpatient curette biopsy.

\section{Case Report}

A 57-year-old Japanese woman (gravida 4 para 4) presented with a 6-month history of light but intermittent postmenopausal vaginal bleeding without abdominal pain. Pelvic examination revealed a 12-mm hypertrophic endometrium via transvaginal ultrasound, and endometrial cytology revealed a few suspicious atypical endometrial cells; however, these findings did not confirm the diagnosis of EC (Fig. 1a). An outpatient endometrial biopsy performed 
with a curette followed by endometrial cytology also suggested that there was not sufficient pathological evidence to diagnose the presence of EC. However, contrast-enhanced magnetic resonance imaging (MRI) of the pelvis showed the disruption of the junctional zone in the posterior wall of the uterus and suggested myometrial invasion of the EC. FIGO stage IB EC was suspected (Fig. 2a, b). The discordance between the findings of the endometrial biopsy and MRI warranted a second endometrial biopsy to obtain histological evidence of EC. The second endometrial biopsy was performed with the aid of an outpatient diagnostic hysteroscopical vision using a 3-mm flexible hysteroscope (Olympus Corporation), but no obviously elevated mass was detected. We observed only irregular gradual eminence on the posterior wall (Fig. 3a). The obtained specimen contained only a small number of fused glands lacking stroma, which was not sufficient to conclusively confirm the presence of malignancy (Fig. 1b).

We subsequently performed an operative hysteroscopy with a resectoscope for tissue sampling under general anesthesia, since we hypothesized that the deep location and the hard consistency of the tumor made endometrial biopsy difficult. An irregularly elevated lesion was observed in the posterior wall of the uterine cavity using an 8.3-mm rigid hysteroscope (Olympus Corporation), and part of this lesion was incised with a loop electrode (Fig. 3b, c). This 5-mm-thick collected tissue included not only endometrium but also adjacent myometrium. Pathological examination of the hysteroscopically incised tissue showed a mixture of well-differentiated endometrioid adenocarcinoma of the uterine body and adenomyosis (Fig. 1c1-c3). This finding indicated the possibility of well-differentiated endometrioid adenocarcinoma of the uterine corpus with adenomyosis or well-differentiated endometrioid adenocarcinoma of the uterine body arising in adenomyosis.

A total of 3 months after her first visit to our hospital, total abdominal hysterectomy and bilateral salpingo-oophorectomy with pelvic/para-aortic lymph node dissection were performed in our patient. Macroscopically, the endometrium was atrophic, and there was no obvious mass in the uterine cavity; however, microscopically, cancer cells were present in the deep myometrium and the final diagnosis was FIGO stage IB, endometrioid adenocarcinoma, G1, diffuse and endophytic type with adenomyosis (Fig. 1d1-d2, 3d1-d2). As adjuvant chemotherapy, 6 courses of paclitaxel and carboplatin (paclitaxel, $175 \mathrm{mg} / \mathrm{m}^{2}$; carboplatin, area under the curve of 6 ) were administered due to deep myometrial invasion of the cancer. The patient has been free from recurrence for over 1 year since laparotomy.

\section{Discussion}

The most frequent malignancy found in cases of postmenopausal bleeding is EC. Diagnosis is usually performed by outpatient-based endometrial biopsy after abnormal findings are detected by transvaginal ultrasound and endometrial cytology. An accurate preoperative diagnosis of endometrial tumor, specifically the presence or absence of malignancy, is important because the extent of surgical treatment is based on staging and pathological tumor characteristics. Surgical treatment for endometrial carcinoma in postmenopausal women generally consists of total hysterectomy and bilateral salpingo-oophorectomy, except for cases in which patients strongly wish to preserve their fertility. Systematic lymphadenectomy would be added to this procedure based on tumor differentiation and depth of myometrial invasion, since the presence of lymph node metastasis is one of the most important prognostic factors in early-stage EC [8]. Additionally, for patients of reproductive age, tumor characteristics preoperatively diagnosed by biopsy are more important, because these patients usually wish to preserve their fertility. 
The most important key point of this case was whether systematic lymphadenectomy should be performed or not. The presence of malignant tumor was strongly suspected by imaging diagnosis using ultrasound examination and MRI, but a precise pathological diagnosis was not achieved by multiple outpatient endometrial tissue biopsies even with the aid of office hysteroscopy. On the other hand, this examination showed solid and irregular gradual eminence on the posterior wall. Therefore, we hypothesized an endophytic tumor growth pattern, and this consistency - derived from the pathological characteristics of adenomyosis existing in the posterior wall of the - uterus might make sampling difficult. Based on this hypothesis, the depth of an operative biopsy was considered necessary to reach the uterine myometrial layer with hysteroscopic resectoscope to examine the relation to the stroma [9]. As a result, through this biopsy using a loop electrode, our diagnosis was in accordance with the final pathological diagnosis after abdominal hysterectomy.

When histopathology of endometrial sampling is inconclusive or inconsistent with the clinical suspicion, diffusion-weighted (DWI) MRI can be used to differentiate the presence of EC over benign disease. Notably, $96 \%$ of all tumors could be identified on fused DWI or T2weighted imaging. In cases where the endometrium is distorted by the presence of leiomyomas or adenomyosis, the restricted diffusion in EC aids tumor detection on DWI, as in this case [10].

The FIGO classification, which was revised in 2008, excluded peritoneal cytology as a factor of staging EC of the uterine body. As for cancer localized within the uterus, the association between the result of peritoneal cytology and patients' prognosis is unknown [11]. In our case, intraoperative peritoneal washing cytology was negative. Although the pressurized distending fluid used in hysteroscopy could cause retrograde dissemination of malignant cells into the peritoneal cavity, it is inconclusive whether this procedure could contribute to the worsened morbidity or mortality among EC patients [12]. Further studies to elucidate the relationship between the usage of diagnostic hysteroscopy and retrograde dissemination are required.

\section{Conclusion}

There are some cases in which gynecologists fail to diagnose EC with outpatient endometrial biopsy technique. Operative biopsy of the uterine endometrium and deep myometrium using a hysteroscopic resectoscope provided us with an accurate diagnosis of uterine endophytic-type EC, in our case, before performing definitive operation namely hysterectomy. Further studies into the utility of operative biopsy, including the deep uterine myometrium, to investigate its efficacy and safety are warranted.

\section{Acknowledgements}

We would like to thank Dr. Akihiko Ozaki for his critical reading of the manuscript.

\section{Statement of Ethics}

The authors have no ethical conflicts to disclose. 


\section{Disclosure Statement}

The authors have no conflicts of interest to declare.

\section{References}

1 Ferlay J, Soerjomataram I, Dikshit R, Eser S, Mathers C, Rebelo M et al. Cancer incidence and mortality worldwide: sources, methods and major patterns in GLOBOCAN 2012. Int J Cancer. 2015 Mar;136(5):E35986.

2 Makker V, Green AK, Wenham RM, Mutch D, Davidson B, Miller DS. New therapies for advanced, recurrent, and metastatic endometrial cancers. Gynecol Oncol Res Pract. 2017 Dec;4(1):19.

3 Creasman WT, Odicino F, Maisonneuve P, Beller U, Benedet JL, Heintz AP et al. Carcinoma of the corpus uteri. J Epidemiol Biostat. 2001;6(1):47-86.

4 Xie B, Qian C, Yang B, Ning C, Yao X, Du Y et al. Risk factors for unsuccessful office-based endometrial biopsy: a comparative study of office-based endometrial biopsy (pipelle) and diagnostic dilation and curettage. J Minim Invasive Gynecol. 2017 Dec;S1553-4650(17)31325-0.

5 Rezk M, Dawood R, Masood A. The safety and acceptability of Pipelle endometrial sampling in premenopausal women in comparison to postmenopausal women with abnormal uterine bleeding. Minerva Ginecol. 2016 Oct;68(5):492-6.

6 Ewies AA, Shaaban KA, Merard R, Zanetto U. Endometrial biopsy in women with abnormal uterine bleeding: inadequate and unassessable categorisation is not clinically relevant. J Clin Pathol. 2014 Aug;67(8):673-7.

7 Mazzon I, Corrado G, Morricone D, Scambia G. Reproductive preservation for treatment of stage IA endometrial cancer in a young woman: hysteroscopic resection. Int J Gynecol Cancer. 2005 SepOct;15(5):974-8.

8 Fares R, Kehoe S, Shams N. Preoperative prediction of lymph nodal metastases in endometrial carcinoma: is it possible?: a literature review. Int J Gynecol Cancer. 2018 Feb;28(2):394-400.

9 Sangle NA, Lele SM. Uterine mesenchymal tumors. Indian J Pathol Microbiol. 2011 Apr-Jun;54(2):243-53.

10 Addley H, Moyle P, Freeman S. Diffusion-weighted imaging in gynaecological malignancy. Clin Radiol. 2017 Nov;72(11):981-90.

11 Pecorelli S. Revised FIGO staging for carcinoma of the vulva, cervix, and endometrium. Int J Gynaecol Obstet. 2009 May;105(2):103-4.

12 Chen J, Clark LH, Kong WM, Yan Z, Han C, Zhao H et al. Does hysteroscopy worsen prognosis in women with type II endometrial carcinoma? PLoS One. 2017 Mar;12(3):e0174226. 


\section{Case Reports in Oncology}
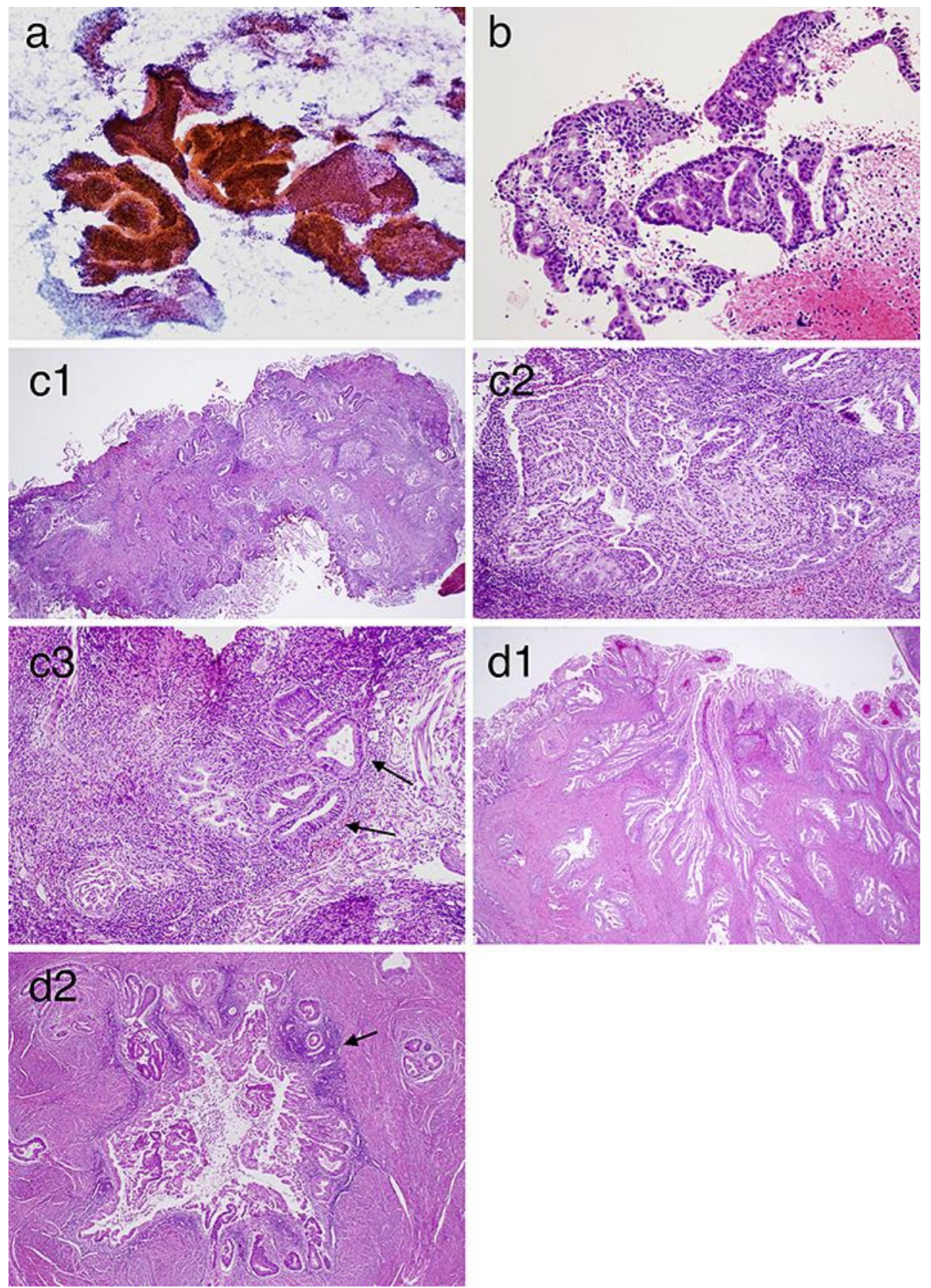

(c) 2018 The Author(s). Published by S. Karger AG, Basel

Honda et al.: Endophytic-Type EC with Adenomyosis Successfully Diagnosed with Hysteroscopic Endometrial Biopsy Using an 8.3-mm Operative Resectoscope

Fig. 1. Endometrial cytological findings and pathological findings of the biopsies and the resected uterus. a Endometrial cytological findings: overlapping cell cluster with inflammatory background and suspected hyperplasia (Pap. staining, magnification $\times 10$ ). b Pathological findings of endometrial biopsy showed fused glands lacking stroma, which was not sufficient to confirm the diagnosis of endometrial cancer (hematoxylin-eosin stain, magnification $\times 20$ ). c Pathological findings by means of hysteroscopic resectoscopy. c1 Distribution of glands in the myometrium (hematoxylin-eosin stain, magnification $\times 2$ ). c2 Abnormal fused gland (hematoxylin-eosin stain, magnification $\times 4$ ). c3 Abnormal glands and adenomyosis (arrows) (hematoxylin-eosin stain, magnification $\times 4$ ). d Final pathological findings of the uterus corpus. d1 Tumorinvaded myometrium from the endometrium (hematoxylin-eosin stain, magnification $\times 2$ ). $\mathbf{d} \mathbf{2}$ Tumor surrounded by adenomyosis tissue (arrow) (hematoxylin-eosin stain, magnification $\times 4$ ) . 


\section{Case Reports in Oncology}
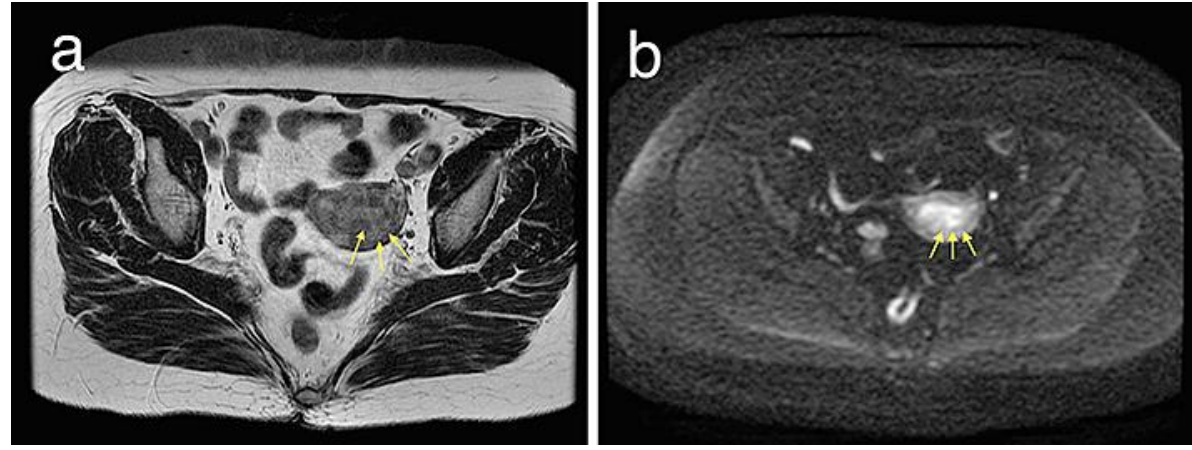

Fig. 2. Findings on contrast-enhanced magnetic resonance imaging. a On T2-weighted imaging, junctional zone was unclear especially on the posterior wall (arrows) and further high intensity from the endometrium area suggested myometrial invasion of the cancer. $\mathbf{b}$ High intensity in diffusion-weighted image and low intensity of apparent diffusion coefficient was consistent with myometrial invasion on T2-weighted imaging (arrows).
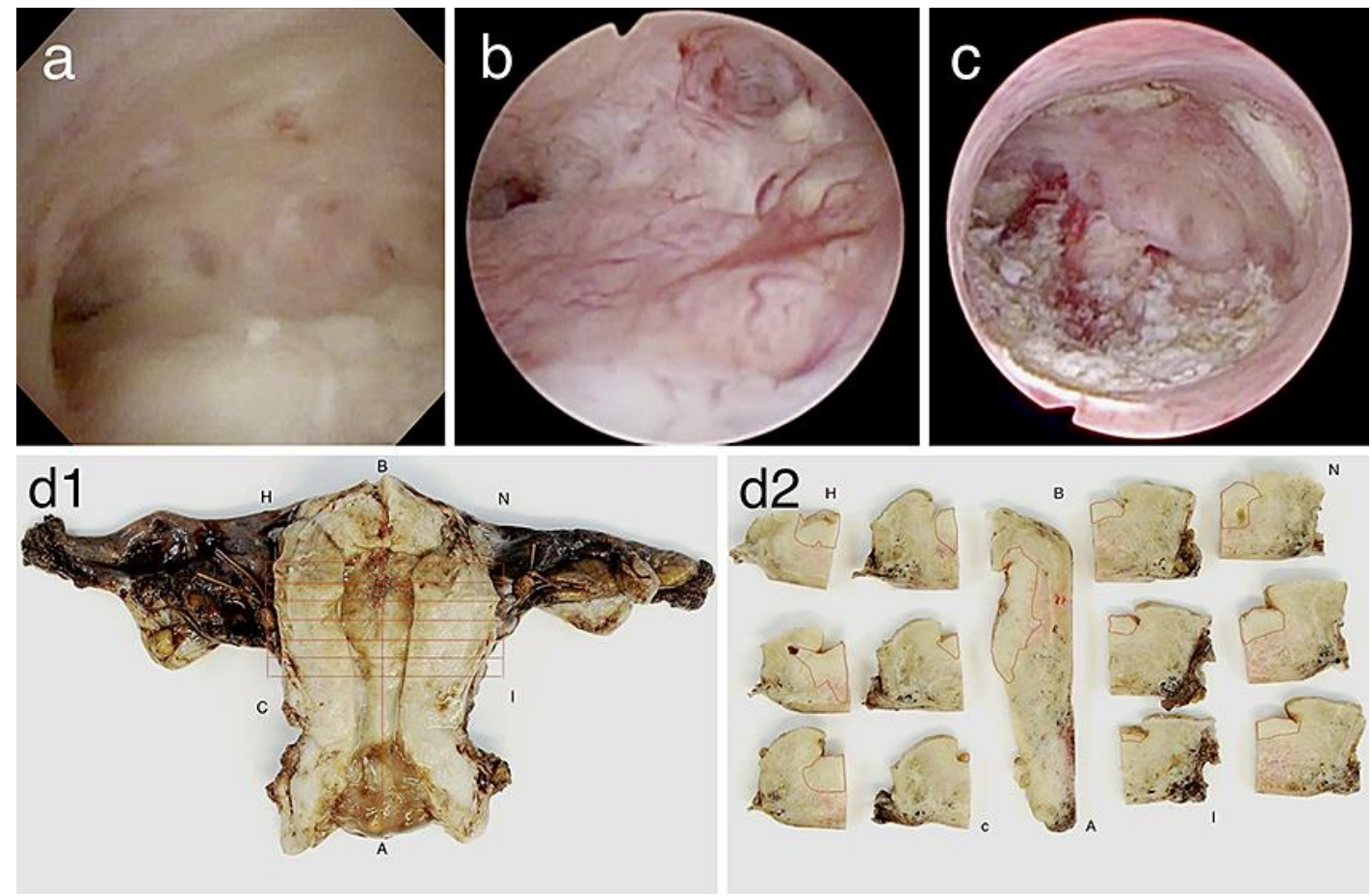

Fig. 3. Hysteroscopic findings and macroscopic findings of the resected uterus and ovaries. a Findings from 3-mm office hysteroscopy: only irregular gradual eminence on the posterior wall without polyps and irregular branching of vessels. b Findings from an 8.3-mm operative hysteroscope before resection of the endometrium and myometrium: irregular branching of vessels was seen on the irregular gradual eminence of the posterior wall and no necrosis was seen. c Findings from an 8.3-mm operative hysteroscope after resection. d1, d2 Macroscopic findings of the resected uterus and ovaries revealed that the tumor was distributed in the posterior wall of the uterus with deep myometrial invasion (within the range of the red line). 